Trinity University

Digital Commons @ Trinity

Psychology Faculty Research

Psychology Department

$1-2016$

\title{
Our Critics Might Have Valid Concerns: Reducing Our Propensity to Conflate
}

Carolyn Becker

Trinity University, cbecker@trinity.edu

Follow this and additional works at: https://digitalcommons.trinity.edu/psych_faculty

Part of the Psychology Commons

Publication Details

Eating Disorders: The Journal of Treatment \& Prevention

\section{Repository Citation}

Becker, C. B. (2016). Our critics might have valid concerns: Reducing our propensity to conflate. Eating Disorders: The Journal of Treatment \& Prevention, 24(1), 79-89. doi: 10.1080/10640266.2015.1113833

This Pre-Print is brought to you for free and open access by the Psychology Department at Digital Commons @ Trinity. It has been accepted for inclusion in Psychology Faculty Research by an authorized administrator of Digital Commons@ Trinity. For more information, please contact jcostanz@trinity.edu. 


\title{
Our critics might have valid concerns: Reducing our propensity to conflate
}

\author{
Carolyn Black Becker, Ph.D.
}

Department of Psychology, Trinity University, San Antonio, TX, USA

Pre-Print: Eating Disorders: The Journal of Treatment and Prevention

Author Information:

Corresponding Author:

Carolyn Black Becker, Ph.D., Department of Psychology, Trinity University, San Antonio, TX, USA. Email: cbecker@trinity.edu

Acknowledgments: The author sincerely thanks Dr. Lisa Smith Kilpela, Dr. Kelly Klump, and Laura Lyster-Mensh for their thoughtful comments and feedback on drafts of this paper.

Disclosure: Dr. Becker serves as Co-Director of the Body Project Collaborative 
As noted by Austin elsewhere in this issue, the field of eating disorders (ED) prevention has made remarkable scientific strides in the past two decades (see Austin, 2016). Over this same period, the field also has seen improved political standing within the greater ED community. For instance, prevention researchers present more regularly at key ED conferences, increasingly via invitation "up on the big stage" in plenaries and keynote addresses. Prevention researchers and advocates also appear to have grown in number and hold more positions in a variety of ranks throughout key ED organizations. Finally, a number of prominent ED researchers who previously held negative opinions about the viability of prevention now support their graduate students and other junior scholars in the pursuit of prevention research.

Despite these positive developments, at times our field still finds itself battling with other members of the ED community (Levine, 2015). For instance, we continue to debate with some community stakeholders (e.g., grass roots advocates consisting of carers and former patients) on a variety of topics including (but not limited to) whether or not ED prevention is possible at all and the degree to which improving body image (on either an individual or community level) will affect the prevalence or onset of EDs. Because many community stakeholders do not publish in academic forums, these debates often emerge at conferences (e.g., Becker, Lyster-Mensch, Banker \& Klump, 2015), on blogs (e.g., Lyster-Mensch, 2015), in private one-on-one discussions, and in communications with advocacy organizations (e.g., IDEACTION, 2015). Members of the ED prevention field have a number of options in engaging in a dialogue with said community stakeholders (and other critics). One option is simply to disengage based on the assumption that resolution is not possible and discourse is a waste of time; there are problems, however, with this approach. For one, we lose potential key allies for dissemination and implementation of evidence-based programs. We also lose the opportunity to understand 
other perspectives, including that of a key ED constituency at a time when engaging with stakeholders is increasingly viewed as critical to advancing improved healthcare (e.g., PCORI, 2014). Further, we lose the opportunity to gain allies who share many common values (e.g., the importance of positive body image) but disagree on the linking to ED prevention. Finally, we may fail to learn if some of their concerns are valid.

Another commonly chosen option is to use the empirical literature to present a different perspective. Indeed, to many prevention researchers, stakeholder comments often seem to indicate a misunderstanding of the subtleties of the literature and the scientific process with regards to prevention. Yet, problems exist with this approach as well. More specifically, in many cases this approach simply does not appear to work. For instance, after a 2014 panel at the International Conference on Eating Disorders aimed (to some degree) at addressing the above issues - a number of stakeholders informed me that the "prevention field just isn't hearing us." Several prevention researchers also reported feeling that stakeholders just didn't "get it." Taking a lesson from Dialectical Behavior Therapy (DBT), it appears that simply debating the empirical literature leaves us in a tug of war that fails to help us find common ground.

A third option, also drawn from DBT, is to search for ways to validate and respond to legitimate critic concerns (i.e., to drop our end of the rope in our game of tug of war). Notably, this involves finding the "kernel of truth" (Linehan, 1993) in critiques of our field and then identifying ways to alter our own behavior. Importantly, this strategy does have a number of disadvantages such as being potentially time consuming (e.g., requires extensive conversations with unhappy stakeholders and/or other critics) and involving self-reflection that can be a rather painful at times. Further, it is unlikely to address all concerns of critics or to fully resolve our differences. Yet, it also offers a critical advantage. Namely, our field's science and influence 
might benefit by addressing legitimate concerns. Further, just as in a DBT therapy session, we might find that validating legitimate concerns has a positive impact on our ability to communicate effectively.

Based on over three years of discussion with community stakeholders, conversations with non-prevention ED researchers and treatment providers, and extensive reflection, I propose that there are two related steps that those of us in the prevention field could take to simultaneously reduce disagreement and improve our science, ability to communicate, and impact. It is important to note that I realize the prevention field consists of a large number of individuals and that we do not all behave in exactly the same way or believe the same things. Moreover, I'm sure some of the points made below have been made elsewhere by someone in prevention before. Lastly, I realize that I have been guilty in the past of the behaviors described below.

\section{Step 1: Stop Conflating Risk Factor Reduction with Eating Disorders Prevention}

Consistent with prevention endeavors for other health problems, ED prevention specialists typically adopt a public health approach to prevention by targeting known or probable modifiable risk factors for EDs (i.e., risk factors that can be manipulated; Kraemer, Kazdin, Offord, Kessler, Jensen, Kupfer, 1997). More specifically, we aim to reduce or prevent risk factors under the assumption that if we can reduce them sufficiently we can decrease the onset of EDs (i.e., prevent EDs) or whatever condition is being studied. Note that there is a permanent bidirectional relationship between risk factor science and prevention science. More specifically, the more we understand about risk factors, the more likely we are to be able to successfully prevent a disorder. It is, however, possible to prevent a disorder without fully knowing the cause. 
For instance, a community can prevent onset of new cases of a contagious disease via quarantine without fully understanding the nature of the disease or even how it is transmitted. On the flip side, for a modifiable risk factor to be deemed causal, we must show that manipulation of said risk factor in fact reduces the onset of EDs (Kraemer et al., 1997). In other words, an ED risk factor by definition is not causal until someone manages to both manipulate it and demonstrate it prevents EDs. To date, almost no risk factors have met this bar.

A critical word in the previous paragraph is the word "assumption" given that most ED "prevention" studies are not designed with sufficient scientific rigor to determine whether or not clinical EDs have been prevented. This is totally understandable for several reasons. First, one needs significant statistical power (i.e., hundreds of participants) to detect a difference in ED onset between an intervention and control groups secondary to the overall low prevalence rate of EDs. One also needs a) a no treatment control condition, b) long follow-up time (e.g., bare minimum of one year and preferably much longer so EDs have time to develop in the control condition), and c) excellent assessment (i.e., gold standard interviewer assessment). All of these equate to significant expense. As a result, and totally consistent with other areas of health research, most studies simply measure reductions in known or probable risk factors including ED symptoms (or what often gets described as disordered eating or ED pathology).

A quick sidebar on the topic of EDs and disordered eating is warranted here as this is one place where some community stakeholders appear to misunderstand the science. Stakeholders in prevention debates often imply that disordered eating is somehow fundamentally different than EDs (e.g., IEDACTION, 2015). In doing so they are, in fact, misinterpreting the distinction between dimensional and categorical assessment. Researchers typically use the term disordered eating to represent dimensional assessment of ED symptomatology (e.g., global scores on the 
Eating Disorder Examination Questionnaire) along the full spectrum of behavior (ranging from completely asymptomatic to those with clinical EDs). The term ED is used to describe those categorical syndromes defined in our diagnostic nomenclatures (i.e., meet DSM 5 criteria). Thus, disordered eating (when fully assessed) encompasses EDs at one end of the spectrum in the same way that "high blood pressure" is included when blood pressure is treated dimensionally in research studies. Use of dimensional measures of disordered eating (or any other risk factor) is often advantageous from a research perspective in that dimensional measures offer both increased statistical power and valid yet inexpensive assessment of ED symptoms; this is the reason disordered eating serves as a proxy for EDs in many research studies, ranging from prevention to genetics. Further, given that subclinical ED symptomatology (which is captured by disordered eating measures) causes pain and suffering (Keel, Brown, Holm-Denoma, \& Bodell, 2011) AND increases risk for clinical EDs (the categorical classification; Jacobi, Hayward, de Zwaan, Kraemer, \& Stewart, 2004), assessing reductions in this part of the dimension also is useful. Yet, we must remember that the medical field typically views "prevention" as being equated to preventing the onset of the categorical condition of interest (i.e., DSM 5 EDs); thus, this is the standard to which our stakeholders/critics are holding us. As a result, if we want to claim that ED prevention works, we must show that we have prevented the onset of clinically significant EDs using categorical assessment. Anything different, including a reduction in dimensional disordered eating is not actually what is commonly meant by prevention.

I suggest that this is not an unreasonable standard for critics to set. For instance, thiamine deficiency is a known direct causal risk factor for the development of Korsakoff Syndrome (KS: Sullivan \& Pfefferbaum, 2008), and alcohol dependence is recognized as the most common pathway for the development of thiamine deficiency (Thompson, 2000). Yet, we would not say 
that a research trial that demonstrated a clear reduction in alcohol dependence had in fact prevented KS without demonstrating a down-the-road reduction in $\mathrm{KS}$, because it might be that the reduction in alcohol dependence (although statistically significant) was insufficient to prevent the endpoint disorder. Instead, we could say that we had reduced a critical risk factor for $\mathrm{KS}$ and that future research could determine if our intervention prevented the endpoint disorder. Importantly, this critique emerges elsewhere in medicine. For instance, researchers and physicians have challenged that existing data do not necessarily support statins in preventing the critical endpoint conditions of myocardial infarction, stroke or death in women (Mora, Glynn, Hsia, MacFadyen, Genest, Ridker, 2010; Rabin, 2014) even though statins lower cholesterol and other risk factors (e.g., hospitalization for unstable angina) in women.

As such, community stakeholders stand on fairly solid ground when they challenge that almost all of our "prevention" studies do not, in fact, provide solid evidence that EDs can be prevented. Moreover, we have only three published trials that provide controlled evidence of true ED prevention effects (Stice et al., 2008; Taylor et al. 2006; Martinsen et al. 2014), and one of these trials (Taylor et al. 2006) only found a onset reduction by examining a specific sub-sample. Further, NO trials have demonstrated a reduction in development of early-onset anorexia nervosa (AN) - which observationally appears to be an ED that often precedes the development of a parent activist; for those with less community stakeholder involvement, parent activists represent some (but not all) of the voices arguing prevention is not possible. In sum, we have a limited number of trials that can be argued to demonstrate true prevention effects, and none of these address the lived experiences of many community stakeholders in that we have not prevented the disorder (early onset AN) that brought such disaster (and even death) into their lives. 
So what do we do? I propose we increase transparency in what we are doing by increasing precision in our language. When we use the phrase "ED prevention" to describe a risk factor reduction trial, we conflate two very different (though related) research methods, and we obfuscate our own understanding of the differences. This in turn makes it appear to outsiders that we think "a" equals "b" even when we know that is not the case. If we consistently label studies incapable of assessing differential ED onset as "risk factor reduction studies" we add clarity to the literature, which is positive in and of itself. Further, it makes it harder for us to pretend our effects are more significant than they are, and might motivate us to identify novel ways to conduct those exceedingly difficult prevention trials. We might also consider referring to our field as the "ED prevention and risk factor reduction field." Although wordy and somewhat unwieldy, the clarity may be worth the extra words.

Step 2: Attempt to (partially) disentangle body image from ED prevention

In addition to conflating prevention of ED with reduction in risk factors, I suggest that we (and community body image activists) are at times guilty of equating improving body image (including body image advocacy efforts) with ED prevention. I realize that one might argue that body image is just a specific example of Step 1. Yet, body image is such a key example, that it deserves some discussion in its own right.

I should note that, as a self-identified body image researcher, I fully acknowledge the relationship between EDs, disordered eating, body dissatisfaction, and thin-ideal internalization. Body dissatisfaction is, in fact, one of the only known causal modifiable risk factors in the development of EDs. Body dissatisfaction not only prospectively predicts onset of some full 
syndrome EDs (Stice, Rohde, Shaw \& Gau, 2011), but manipulation of body dissatisfaction also resulted in decreased onset of EDs relative to a control group (Stice, 2008), which is the critical finding needed to label a modifiable risk factor as causal (Kraemer et al., 1997). Further, evidence suggests that no cases of bulimia nervosa $(\mathrm{BN})$ have been found without exposure to Western influence and the presumable influence of the thin-ideal (Keel \& Klump, 2003). Also, although evidence suggests that AN can arise in the absence of Western culture and body image concerns (Keel \& Klump, 2003), increased rates of AN have been linked to thinness idealization (Hoek et al., 2005). Thus, it seems safe to conclude that body dissatisfaction is a causal risk factor for at least some EDs, which makes it an appealing target for prevention efforts. Further, because body dissatisfaction is widespread and causes misery even in those who never develop EDs, other community stakeholders (e.g., schools) find reducing body dissatisfaction reasonably attractive. Body dissatisfaction also appears to be a risk factor for other undesirable conditions including depression and suicidality (Bearman, Martinez, \& Stice, 2006; Crow, Eisenberg, Story, \& Neumark-Stzainer, 2008; Rawana , 2013). Finally, decreasing negative body image environments more broadly may be helpful for those recovering from an ED. Research suggests that failure to address body image disturbance in those with EDs increases risk for relapse (Keel, Dorer, Franko, Jackson, \& Herzog; 2005), and patients often look to the environment for evidence to support negative body image beliefs. In summary, there are many reasons to target body image if one wants to prevent EDs and ED relapse.

Despite these benefits, there also are some downsides to linking body dissatisfaction with EDs prevention. These downsides are not sufficient to support the proposition that our field abandon its focus on body image. However, they are sufficient to suggest that being more careful 
with our language, more nuanced in our claims, and more assertive in helping community activists increase their accuracy might be beneficial to our field.

For instance, there is no evidence to suggest that body dissatisfaction plays a triggering role in all EDs. More specifically, we have no compelling evidence that body dissatisfaction is a causal risk factor for many cases of AN. As noted above, body dissatisfaction is not a requirement for the development of AN (Keel \& Klump, 2003). As a result, when we too strongly proclaim the link between body dissatisfaction and EDs (or fail to check community/political body image activists and the media when they do the same), we invalidate the lived experiences of a sizeable number of patients, recovered individuals, carers, and even front line treatment providers. As any DBT therapist will tell you, invalidation is a highly effective strategy for starting a communication-losing tug of war. If we simply hold fast to our position (i.e., fail to drop our end of the rope), we end up baffled by their anger and seeming denial of the scientific support for our standpoint; they in turn view us as disregarding other important and scientifically-supported perspectives. We all lose time and energy in the fight.

To improve this situation, I suggest we strive for increased accuracy. For instance, we could routinely state that body dissatisfaction is a risk factor for some EDs as opposed to stating categorically that body dissatisfaction increases ED risk. Although the latter technically is accurate from a scientific perspective, it implies that all EDs are caused by body dissatisfaction, which is not accurate. Further, since body dissatisfaction likely plays a differential role in different EDs, grouping all forms of EDs together when studying body dissatisfaction may hinder scientific progress by obscuring important questions about differences.

In addition, we could note the importance of other risk factors by reminding listeners/ readers that EDs are caused by a complex interplay of biological, psychological, and 
environmental factors - before discussing body dissatisfaction. In other words, explicitly lay out a biopsychosocial model as opposed to implying a purely sociocultural perspective. Further, we can push activists and the media to adopt more careful language and to avoid making statements such as "body dissatisfaction leads to eating disorders" which suggests a simplistic association. We may not succeed, but we can try to make sure we (and others) do not overstate relationships. Another concern raised by some community stakeholders is that linking body image to EDs increases stigma of EDs by contributing to the perception that EDs are trivial problems of vanity. Unfortunately, there is likely some truth to this concern. Indeed, the ED field has sought to describe EDs as serious biologically-based disorders (Klump, Bulik, Kaye, Treasure \& Tyson, 2009) in part to counter stigmatizing perceptions of EDs as frivolous disorders of little rich girls who don't like their bodies. Many community stakeholders also have embraced biologicallybased messaging for a variety of reasons. Given that we live in a Cartesian culture that assumes the body is more real than the mind (Kirmayer \& Looper, 2007), one can appreciate the appeal of biologically-based language even if data suggest it is unlikely to fully succeed at reducing stigma or achieving other goals (see Deacon, 2013 for discussion of this in the broader mental health field). Unfortunately, when we overly link sociocultural body image concerns back to EDs, we run directly counter to biological messaging, thus setting the stage for conflict.

Although there is no perfect fix for this conundrum (and I do not support throwing in the proverbial towel on this one), there are several options for trying to mitigate the situation. First, we can work to broaden the linking of body image to other mental and physical health concerns (e.g., depression, suicidality, anxiety (including social anxiety), smoking, physical activity, consumption of nutrient dense diet, sleep, overall quality of life). There is ample data to suggest that body image disturbance is no different than smoking in that it is a non-specific risk factor for 
a host of negative outcomes. Yet, as one non-prevention researcher noted during the discussion portion of my 2012 keynote address at the Australian and New Zealand Academy of Eating Disorders meeting, to many in the ED community, it appears that body image researchers/activists use "EDs as a cudgel to convince the rest of the world that body image matters" (note that plenty in the audience seemed to agree). Making a broader case for the importance of healthy body image, thus, might simultaneously a) improve the perception of body image as important, b) provide access to increase funding sources, and c) reduce the perceived downward pull of body image on EDs when it comes to stigma.

A second option is to link body image and EDs only when strategic for promoting our programs. For instance, at this point, many communities have begun to recognize that body image is a problem in and of itself. As a result, in many cases, there is no need to bring in the ED connection when delivering body image programs, which makes it easier for some skeptical community stakeholders to suddenly become partners. For instance, when disseminating the Body Project, a body image program that has been shown to reduce the onset of EDs in one trial, we now encourage university partners to not mention EDs. Instead, they simply promote the Body Project as an empirically supported program for improving body image. One advantage is that it makes the program more relevant to a larger percentage of the population. Thus, the Body Project becomes, first and foremost, something good for many university students that (as an aside) might prevent some EDs (but if it doesn't - it's still good for other students). Interestingly, this approach appears to make it easier to recruit students to participate in groups. Further, I've seen skeptical stakeholders subsequently promote the Body Project and become allies of sorts.

Similarly, when DOVE and the World Association of Girl Guides and Girl Scouts (WAGGGS) decided to adopt the Body Project as the basis for a new body confidence program, 
they chose to eschew any discussion of EDs in its promotion. This served two purposes. One, it remains unclear whether or not the program will prevent EDs when disseminated on a global scale - so pursuant to Step 1, they avoided overly grand claims. Second, as with many universities, DOVE/WAGGS want to address problems that affect a large percentage of their population. Because EDs only occur in a minority of girls, preventing EDs doesn't directly meet the main goals of this organization. In contrast, body image is an endpoint problem for a large population of girls - not merely a stepping stone to a bigger problem for a smaller number of girls. Further, if in fact, the Body Project did prevent a few EDs along the way, then this is just an added benefit. In summary, perhaps surprisingly, it appears easier to disseminate programs that were originally conceived of as prevention programs under "body image improvement" branding, and it helps find more common ground with some community stakeholders.

A final possible solution, is to expand the range of probable risk factors we try to address with prevention programs. Currently, it appears as though an overwhelming majority of efforts directed at ED prevention target body image. This has the unintended effect of suggesting that we think the only way to prevent EDs is via body dissatisfaction and that body dissatisfaction is the singular most important risk factor for EDs. If we expand the range of possible targets, then our focus on body image remains but becomes less dominant. It is beyond the scope of this paper to identify other targets, but I encourage us to look for research and reviews that consider the full biopsychosocial spectrum (e.g., see Culbert, Racine, \& Klump, 2015).

\section{Summary}

To conclude, the ED prevention field has made remarkable progress over the past two decades. Despite this, we continue to find ourselves at odds with some members of the broader ED community. Although we can choose to ignore our critics or simply debate them, it may be 
more effective to identify areas where we can understand the legitimacy of their concerns and seek to alter our approach. The suggested changes in our behavior that I propose include: 1) increasing transparency by consistently labeling studies as "risk factor reduction" studies instead of "prevention" studies when they reduce risk factors (including dimensionally assessed disordered eating) versus showing reductions in categorical EDs in a controlled trial, 2) increase precision in our language regarding the connection between body image and EDs by noting that body dissatisfaction is a risk factor for some (but not all) EDs, 3) more explicitly promote the biopsychosocial perspective in our presentations and publications versus implicitly (or explicitly) promoting a sociocultural model, 4) more regularly connect body image to a range of negative outcomes beyond EDs, and 5) reduce, if not eliminate, discussion of EDs when promoting programs that target body image. Although these steps are unlikely to resolve all differences, they may increase both our impact and our ability to effectively communicate with key ED constituencies. 


\section{References}

Austin, S. B (2016). The last word: Accelerating progress in eating disorders prevention: A call for policy translation research and training. Eating Disorders: Journal of Treatment and Prevention

Basso, C., Perazzolo Marra, M., Rizzo, S., De Lazzari, M., Giorgi, B., Cipriani, A., ... Illiceto, S. (2010). Arrhythmic mitral value prolapse and sudden cardiac death. Circulation, 121(9), 556-566. doi: 10.1161/CIRCULATIONAHA.109.906479

Bearman, S.K., Martinez, E., \& Stice, E. (2006). The skinny on body dissatisfaction: A longitudinal study of adolescent girls and boys. Journal of Youth Adolescence, 35(2), 217-229.

Becker, C. B., Lyster-Mensh, L. C., Banker, J., \& Klump, K. (2015, April). Difficult dialogues in eating disorders: Can we use AED relationships to learn from our diversity and advance our field even when we disagree? Workshop presented at the 2015 International Conference on Eating Disorders, Boston, MA.

Crow, S., Eisenberg, M.E., Story, M., \& Neumark-Sztainer, D. (2008). Suicidal behavior in adolescents: Relationship to weight status, weight control behaviors, and body dissatisfaction. International Journal of Eating Disorders, 41(1), 82-87.

Culbert, K. M., Racine, S. E., \& Klump, K. L. (2015). Research review: What we have learned about causes of eating disorders - a synthesis of sociocultural, psychological, and biological research. Journal of Child Psychology and Psychiatry. Advance online publication. doi: 10.1111/jcpp.12441 
Deacon, B. J. (2013). The biomedical model of mental disorder: A critical analysis of its validity, utility, and effects on psychotherapy research. Clinical Psychology Review, 33(7), 846861.

Hoek, H. W., Van Son, G. E., van Hoeken, D., Bartelds, A. I. M., \& Van Furth, E. F. (2005). Changes in the incidence of eating disorders. Paper presented at the International Conference on Eating Disorders, Montreal.

International Eating Disorders Action (2015, June 11). Letter to the Butterfly Foundation. Retrieved at: http://internationaleatingdisorderadvocacy.blogspot.ie/2015/06/letter-tobutterfly-foundation.html?m=1.

Jacobi, C., Hayward, C., de Zwann, M., Kraemer, H. C., \& Stewart Agras, W. (2004). Coming to terms with risk factors for eating disorders: Application of risk terminology suggestions for general taxonomy. Psychological Bulletin, 130(1), 19-65.

Keel, P. K., Brown, T. A., Holm-Denoma, J., \& Bodell, L. P. (2011). Comparison of DSM-IV versus proposed DSM-5 diagnostic criteria for eating disorders: Reduction of eating disorder not otherwise specified. International Journal of Eating Disorders, 44(6), 553560.

Keel, P. K., Dorer, D. J., Franko, D. L., Jackson, S. C., \& Herzog, D. B. (2005). Postremission predictors of relapse in women with eating disorders. American Journal of Psychiatry, 162(12), 2263-2268.

Keel, P. K. \& Klump, K. L. (2003). Are eating disorders culture-bound syndromes? Implication for conceptualizing their etiology. Psychology Bulletin, 129(5), 747-769. 
Kirmayer, L. J., \& Looper K. J. (2007). Somatoform disorders. In Hersen, M. Turner, S.M., \& Beidel, D. C. (Eds.), Adult Psychopathology and Diagnosis $5^{\text {th }}$ Edition (pp. 410-472). Hoboken, NJ: John Wiley \& Sons.

Klump, K. L., Bulik, C. M., Kaye, W. H., Treasure, J., \& Tyson, E. (2009). Academy for eating disorders position paper: eating disorders are serious mental illnesses. Int J Eat Disord, 42(2), 97-103.

Kraemer, H. C., Kazdin, A. E., Offord, D. R., Kessler, R. C., Jensen, P. S., \& Kupfer, D. J. (1997). Coming to terms with the terms of risk. Archives of General Psychiatry, 54, 337343.

Levine, M. P. (2015, March 2). Does prevention work (and is this even a fair question)? Gürze-Salucore Online Catalogue. Retrieved from http://www.edcatalogue.com/doesprevention-work/

Linehan, M. (1993). Cognitive-Behavioral Treatment of Borderline Personality Disorder. New York, NY: Guilford Press.

Lyster-Mensh, L. C., (2015, April 7). Do we know enough about eating disorders to prevent them? Retrieved from: http://www.laurassoapbox.net/2015/04/do-we-know-enoughabout-eating.html\#comment-form

Martinsen, M., Bahr, R., Borrensen, R., Holme, I., Pensgaard, A. M., \& Sundgot-Borgen, J. (2014). Preventing eating disorders among young elite athletes: A randomized controlled trial. Medicine \& Science in Sports \& Exercise, 46(3), 435-447.

Mora, S., Glynn, R. J., Hsia, J., MacFadyen, J. G., Genest, J., \& Ridker, P. M. (2010). Statins for the primary prevention of cardiovascular events in women with elevated high-sensitivity c-reactive protein or dyslipidemia. Results from the justification for the use of statis in 
prevention: An intervention trial evaluating rosuvastitin (JUPITER) and meta-analysis of women from primary prevention tools. Circulation, 121, 1069-1077.

Patient Centered Outcomes Research Institute (2014, September 10). Why PCORI was created. Retrieved from: http://www.pcori.org/about-us/why-pcori-was-created

Rabin, R. C. (2014, May 5). A new women's issue: Statins. Retrieved from: http://well.blogs.nytimes.com/2014/05/05/a-new-womens-issue-statins/

Rawana, J.S. (2013). The relative importance of body change strategies, weight perception, perceived social support, and self-esteem on adolescent depressive symptoms: Longitudinal findings from a national sample. Journal of Psychosomatic Research, 75 (1), 49-54.

Stice, E., Marti, C. N., Spoor, S., Presnell, K., \& Shaw, H. (2008). Dissonance and healthy weight eating disorder prevention programs: Long-term effects from a randomized efficacy trial. Journal of Consulting and Clinical Psychology, 76(2), 329-340.

Stice, E., Rohde, P., Shaw, H., \& Gau, J. (2011). An effectiveness trial of a selected dissonancebased eating disorder prevention program for female high school students: Long-term effects. Journal of Consulting and Clinical Psychology, 79(4), 500-508.

Sullivan, E. V., \& Pfefferbaum, A. (2009). Neuroimaging of the Wernicke-Korsakoff syndrome. Alcohol and Alcoholism, 44(2), 155-165.

Taylor, C. B., Bryson, S., Luce, K. H., Cunning, D., Doyle, A. C., Abascal, L. B., Rockwell, R., Dev, P., Winzelberg, A. J., \& Wilfley, D. E. (2006). Prevention of eating disorders in atrisk college-age women. Archives of General Psychiatry, 63(8), 881-888.

Thompson, A. D. (2000). Mechanisms of vitamin deficiency in chronic alcohol misusers and the development of Wernicke-Korsakoff syndrome. Alcohol and Alcoholism, 35, 2-7. 